\title{
Research Article \\ Delineation of Crystalline Extended Defects on Multicrystalline Silicon Wafers
}

\author{
Mohamed Fathi \\ Silicon Technology Unit of Algiers (UDTS), 2 Boulevard Frantz Fanon, BP 399 Alger-Gare, Algiers, Algeria
}

Received 23 March 2006; Accepted 15 August 2006

Recommended by Nicolas Alonso-Vante

We have selected Secco and Yang etch solutions for the crystalline defect delineation on multicrystalline silicon (mc-Si) wafers. Following experimentations and optimization of Yang and Secco etching process parameters, we have successfully revealed crystalline extended defects on mc-Si surfaces. A specific delineation process with successive application of Yang and Secco agent on the same sample has proved the increased sensitivity of Secco etch to crystalline extended defects in mc-Si materials. The exploration of delineated mc-Si surfaces indicated that strong dislocation densities are localized mainly close to the grain boundaries and on the level of small grains in size (below $1 \mathrm{~mm}$ ). Locally, we have observed the formation of several parallel dislocation lines, perpendicular to the grain boundaries. The overlapping of several dislocations lines has revealed particular forms for etched pits of dislocations.

Copyright (c) 2007 Mohamed Fathi. This is an open access article distributed under the Creative Commons Attribution License, which permits unrestricted use, distribution, and reproduction in any medium, provided the original work is properly cited.

\section{INTRODUCTION}

The presence of crystalline defects and impurities in multicrystalline silicon (mc-Si) wafers leads to loss of energetic efficiency of the photovoltaic cells $[1,2]$. This is due to the degradation of the electrical properties which are correlated with the crystalline defect density in the bulk material $[3,4]$. Indeed, crystalline defects create recombination centers, and consequently reduce lifetime of minority charge carriers. The aim of this paper is the setting in of a specific delineation process for crystalline extended defects in the case of mc-Si bulk and the study of their propagation. This developed technique will permit us to qualify mc-Si ingots grown at our laboratory (UDTS) and compare their characteristics to others produced by major mc-Si producers. Observation with a scanning electron microscope (SEM) of the crystalline extended defects (dislocations, stacking faults, twins, precipitates, etc.) requires a chemical etching operation known as delineation process [5]. Delineation consists of the action of a selective etching agent which is able to etch more quickly the zones of the crystalline defects than the perfect crystalline zones. This is due to the fact that in region of defects, the disturbance of the crystal lattice causes weak atomic bonds. The delineation of the defects takes place only on the crystal grain level; the zones of grain boundaries are uniformly and more quickly etched because atomic bonds are weaker there [2]. Chemical delineation is followed by SEM observation. Then, identification and counting of the defects per unit area are carried out.

Several chemicals for defect delineation such as Dash, Sirtl, Secco, Yang, Wright have been validated and are used for monocrystalline silicon defect analysis. The issue of defects delineation process depends on silicon surface crystallographic orientation and surface topography [6]. In order to perform the delineation process for $\mathrm{mc}$-Si material where there are multiple crystallographic orientations, it will be necessary to select the more sensitive chemical solution to defects and adjust its application conditions (time, agitation, temperature, etc.). Historically, Dash etch was the first used. It reveals dislocations in all crystallographic orientations but necessitates very long etching times [7]. Sirtl reveals dislocations only on (111) surfaces [8]. Secco etches defects in all orientations and exposes circular defect pits [9]. Yang solution gives good defect delineation in all orientations and its etch-pits shapes (triangular, quadratic, etc.) are functions of surface orientation $[10,11]$. Wright is considered to be the finest chemical defect delineation solution, specially for detection of induced defects by hot processing. It is acting in all orientations but its composition is more complex than Secco and Yang. Furthermore, Wright etch is less sensitive to 
TABle 1: Yang and Secco parameters effects on etched dislocation pits of mc-Si samples.

\begin{tabular}{lccccc}
\hline Sample reference & Solution & Time $(\mathrm{min})$ & Pit shape & Width $(\mu \mathrm{m})$ & Decorating effect \\
\hline Y0 & Yang & 0.5 & Too small & 0.5 & Weak \\
Y1 & Yang & 1 & Triangular/quadratic & 1 & Good \\
Y2 & Yang & 2 & Triangular/quadratic & 2 & Good \\
Y2u & Yang & $2+$ Ultrasonic & Overetching & $4-6$ & Medium \\
Y3 & Yang & 3 & Triangular/quadratic & 3.8 & Good \\
Y4 & Yang & 4 & Overetching & Irregular & Medium \\
Y5 & Yang & 5 & Overetching & Irregular & Bad \\
S0 & Secco & 0.5 & Too small & 0.6 & Weak \\
S1 & Secco & 1 & Circular & 1.1 & Good \\
S2 & Secco & 2 & Circular & 2.3 & Good \\
S2u & Secco & $2+$ Ultrasonic & Circular & 4 & Good \\
S3 & Secco & 3 & Circular & 3.3 & Good \\
S4 & Secco & 4 & Circular & 4.6 & Good \\
S5 & Secco & 5 & Overetching & Irregular & Medium \\
\hline
\end{tabular}

dislocations generated during crystal growth than Secco and Yang solutions $[11,12]$. Because of our interest in studying the dislocations induced during HEM mc-Si growth, and due to the different crystallographic grain orientations on this material, we have chosen to develop our defect analysis process with Secco and Yang etching solutions. Effectively, these two solutions are sensitive to all kinds of defects and also to all crystallographic orientations. However, the specificity of mc-Si material will necessitate a special adjusting of Yang and Secco etching process parameters in order to have the sharpest etch pits and the cleanest surfaces for SEM analysis.

\section{EXPERIMENTAL}

We have used P-type (Boron-doped) mc-Si wafers with electrical resistivity about $1 \Omega \mathrm{cm}$. These wafers of $10 \times 10 \mathrm{~cm}^{2}$ area were cut from ingots grown at our laboratory by the heat exchanger method (HEM) [13]. The first step was thinning and polishing the as-cut mc-Si wafers in order to remove the saw damage and slurry residue on the surface. During this step, we used a polishing solution made by mixing $\mathrm{HNO}_{3} / \mathrm{CH}_{3} \mathrm{COOH} / \mathrm{HF}$ with $(5: 3: 2)$ concentrations. After 2 minutes of etching time, we rinsed thoroughly the mc-Si material with deionized water and dried it under a nitrogen gun. To test the Secco and Yang solutions, we cut 14 samples from an mc-Si polished wafer. These samples were referenced as shown in Table 1. We have varied mainly the etching time and agitation mode. Note that just a few minutes before each delineation process, the native $\mathrm{SiO}_{2}$ formed at the surface was etched by immersing the samples in diluted HF (10\%) solution for 30 seconds followed by rinsing with deionized water and drying in nitrogen. The mc-Si samples were then ready for the defect delineation trials and analysis.

The Secco $[6,9]$ formulation is $\mathrm{HF} / \mathrm{K}_{2} \mathrm{Cr}_{2} \mathrm{O}_{7} / \mathrm{H}_{2} \mathrm{O}$ obtained by mixing 2 parts of $\mathrm{HF}$ with 1 part of $\mathrm{K}_{2} \mathrm{Cr}_{2} \mathrm{O}_{7} / \mathrm{H}_{2} \mathrm{O}$ at $(0.15 \mathrm{M})$ or $\left(44 \mathrm{~g} \mathrm{~K}_{2} \mathrm{Cr}_{2} \mathrm{O}_{7}\right.$ in 1 litre of $\left.\mathrm{H}_{2} \mathrm{O}\right)$. The Yang $[6,10]$ formulation is $\mathrm{HF} / \mathrm{CrO}_{3} / \mathrm{H}_{2} \mathrm{O}$ obtained by mixing 1 part of $\mathrm{HF}$ with 1 part of $\mathrm{CrO}_{3} / \mathrm{H}_{2} \mathrm{O}$ at $(1.5 \mathrm{M})$ or $(150 \mathrm{~g}$ $\mathrm{CrO}_{3}$ in 1 litre of $\left.\mathrm{H}_{2} \mathrm{O}\right)$. After the Secco or Yang delineation, the samples were immediately rinsed in water and dried in nitrogen. Then, SEM observations of the treated samples were carried out.

\section{RESULTS AND DISCUSSION}

Table 1 summarizes the etching parameters that were varied during Yang and Secco delineation studies. Generally, a dipping time between 1 and 2 minutes gives good defect delineation and we clearly distinguish dislocations, grain boundaries, twins, and slip lines. Our experiments confirm the revelation of crystalline defects with the Yang solution starting from 30 seconds of dipping time. However, for 30 seconds, the dislocation pits are too small $(0.5 \mu \mathrm{m})$ to allow any fast exploration with SEM at low magnifications. Experiments with the Yang etch showed that a time of immersion from 1 to 2 minutes without ultrasonic agitation gives the best results. The shapes of Yang-etched pits depend on the crystallographic orientation of the mc-Si grains and are mainly triangular or quadratic. After immersion in Yang solution for 2 minutes with ultrasonic agitation, etching becomes too active, leading to defect-etched pits which are too broad and irregular in dimension. To conclude, the study of the Yang solution indicates that a time of immersion from 1 to 2 minutes without ultrasonic agitation provides the best results. The same study conducted with Secco etching process showed that the sharpest defect delineation was obtained with a dipping time between 1 and 2 minutes. However, in Secco process, the ultrasonic agitation is very useful for the elimination of gas bubble artifacts and results in circular dislocation pits. Thus, the recommended process with Secco etch is from 1 to 2 minutes of dipping time with ultrasonic agitation.

Figures 1 and 2 are good illustrations of Secco and Yang defect delineation processes under the optimized conditions given above. We have clearly delineated dislocations, twins, grain boundaries, and dislocation lines. We have noticed that zones close to the grain boundaries are most favorable for the revelation of strong populations of defects. The exploration 


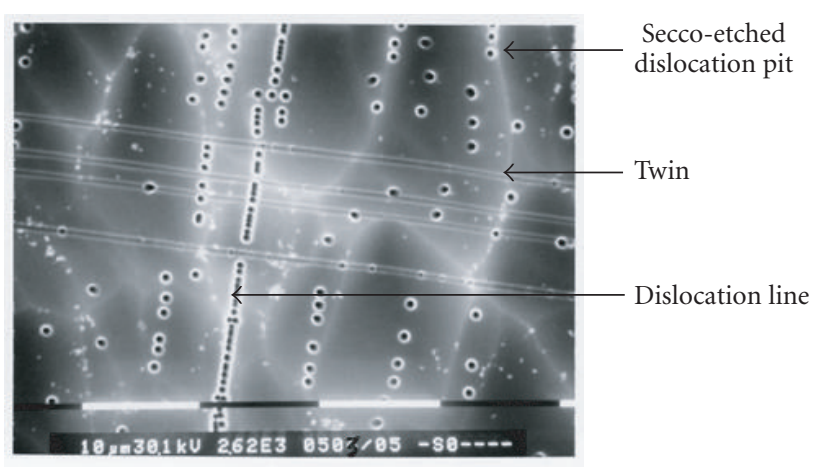

FIgUre 1: Crystalline defects delineated by Secco etch on mc-Si.

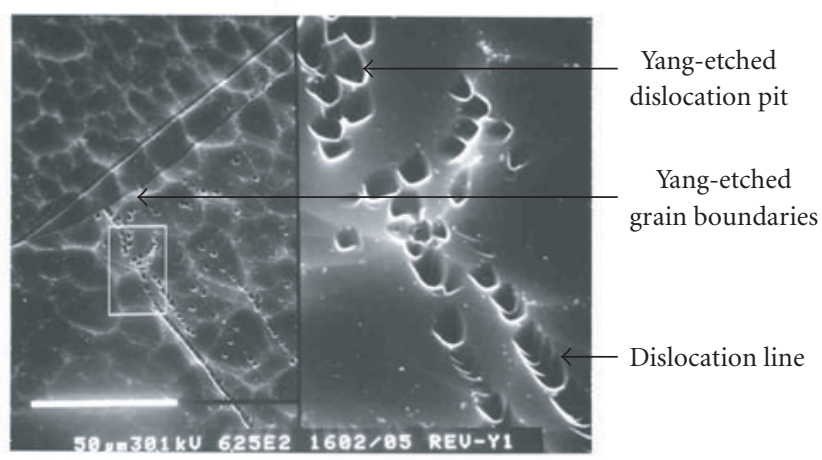

Figure 2: Crystalline defects delineated by Yang etch on mc-Si.

of the surface indicates that zones with high dislocation densities are localized mainly close to the grain boundary zone on the level of the small grain sizes (below $1 \mathrm{~mm}$ ). This phenomenon is illustrated by Figure 3 and it is foreseeable, since on the level of the grain boundary there is a change of the crystallographic orientation leading to a zone of stress which finds its thermodynamic equilibrium by the emission of dislocations [14]. Locally, we have noted the formation of several parallel dislocation lines which are perpendicular to grain boundary (see Figure 4). This observed arrangement of parallel dislocation lines is indicator of dislocation loops created during mc-Si ingot growing process and is principally depending on cooling conditions.

In order to compare the action of dislocation localization between Secco and Yang solutions, we carried out a Secco revelation on a sample revealed before with Yang and vice versa. For this, we initially took the Y2 sample (revealed by Yang 2 minutes) and then we added to it a Secco revelation for 1 minute with ultrasonic agitation. We observed that all initially triangular defects are transformed into circles (see Figure 5), which implies that Secco marks all the places already marked with Yang. But we remark some new zones again (nonexistent before) as small circles (inferior to $1 \mu \mathrm{m}$ ) which means that the Secco solution decorates defect zones which were not previously localized with Yang. In the same way, we carried out the opposite operation by taking the S2 sample (revealed by Secco for 2 minutes) and we applied to it the Yang etch for 1 minute. All the circular zones were

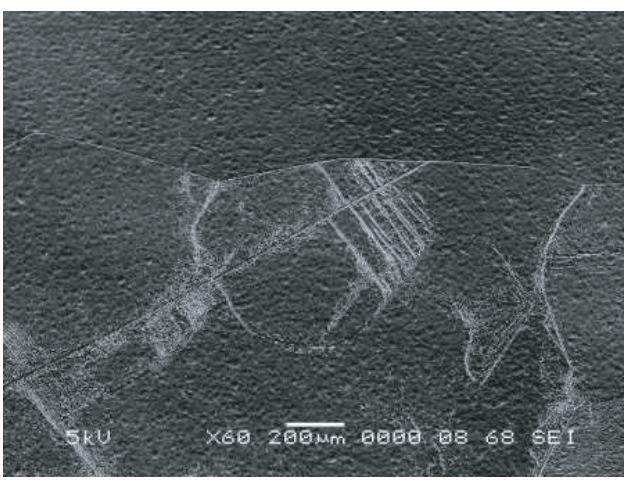

FIGURE 3: Defect-free surface nearby zones with high dislocation densities.

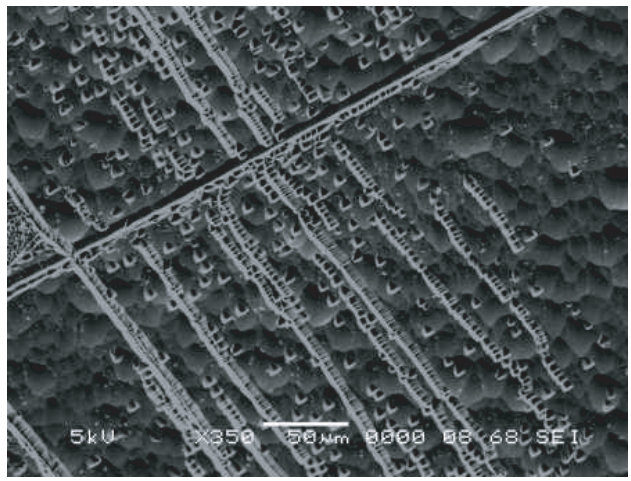

FIGURE 4: Formation of dislocation lines perpendicular to the grain boundaries.

truncated by the Yang action and we obtained the formation of right pit edges. However, we did not find newly revealed zones (not more than $1 \mu \mathrm{m}$ ) with the Yang solution, which makes it possible to state that Yang etch did not delineate new defected areas in opposite to Secco etch. The two preceding tests show that the sensitivity of Secco to crystalline defects is higher than that of Yang etch. This consolidates our choice of Secco for the calculation dislocation density on mc-Si materials.

It is of great importance to study the distribution of crystalline defects throughout the cross-section of mc-Si bulk and the influence of the process parameters. Then, we have observed a section of mc-Si revealed with the Secco solution during 2 minutes. We choose a sample with a section corresponding to a natural cleaving plane, in order to have a very smooth surface for SEM observation. We explored the entire cross-section of this sample and we noticed some particular defect shapes due to the overlapping of several dislocations and according to various angles to the same spot (see Figure 6). However, nothing seems to indicate the presence of stacking fault defects. According to the literature [11], it will be more probable to obtain stacking faults shapes after a high-temperature treatment such as oxidation or diffusion process that the mc-Si substrate will undergo during cell fabrication. Finally, by using Secco delineation process, we have compared the dislocation density of our mc-Si to other mc-Si materials produced by major photovoltaic companies. These parameters are summarized in Table 2 and indicate that in 
TABLE 2: Comparative study of dislocation density values from other mc-Si producers.

\begin{tabular}{lc}
\hline mc-Si Company & Dislocation density $\left(\mathrm{cm}^{-2}\right)$ \\
\hline Bayer-BAYSIX & $3 \cdot 10^{5}$ \\
Solarex-SOLAREX & $10^{4}-10^{6}$ \\
Sumitomo Sitix-EMC & $10^{6}-10^{8}$ \\
UDTS HEM & $10^{5}-10^{6}$ \\
\hline
\end{tabular}

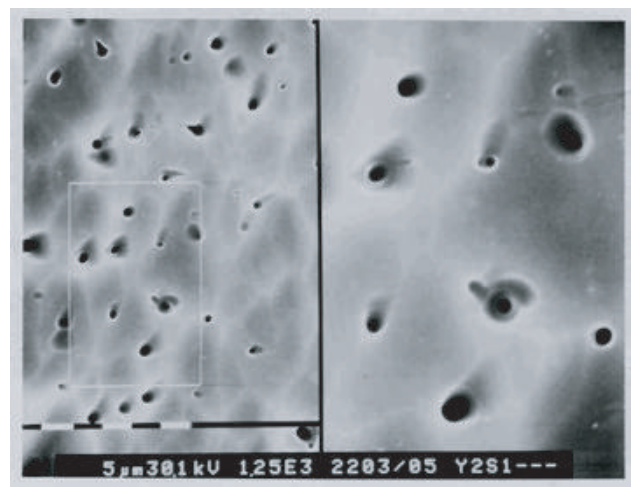

FIGURE 5: Secco solution detects new defected zones of mc-Si sample initially Yang-etched.

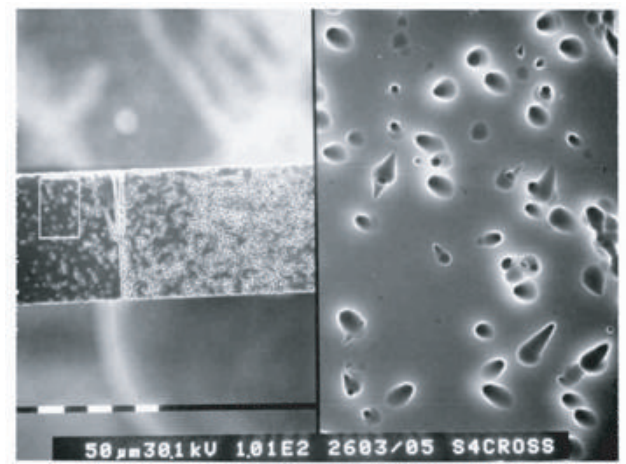

FIGURE 6: Various pit shapes due to the overlapping of several dislocation lines.

terms of dislocation densities, our samples are of comparable quality with respect to the mc-Si materials fabricated by other companies.

\section{CONCLUSION}

We have successfully optimized Secco and Yang etching parameters for crystalline defect delineation on mc-Si material. We have clearly distinguished dislocations, grain boundaries, twins, and dislocation lines. The shapes of Yang etch pits are triangular or quadratic according to crystallographic orientation on the grain level. We have noticed that in Secco etching process, the dislocation pits are circular. A comparative study of these two etches proved that Secco etch is more sensitive to crystalline defects than Yang etch in the case of mc-Si surfaces. We have observed some important phenomena specific to the mc-Si HEM material, like the concentration of dislocations in the vicinity of grain boundaries and irregular repartit ion of these defects from grain to grain. Cross-sectioning experiments using Secco solution clearly revealed crystalline defects which give us a good tool for analysis of crystalline defects through bulk material. This technique will be very helpful for studding process impact on defects propagation in mc-Si bulk material and also for performance and quality enhancing.

\section{ACKNOWLEDGMENTS}

This work was realized at Silicon Technology Development Unit (UDTS), and was supported by the Algerian Ministry of Higher Education and Scientific Research (MESRS), Algiers, Algeria. I would like to thank D. Bouhafs, L. Haddada, A. Manseri, and Y. Belaroussi for their technical support.

\section{REFERENCES}

[1] M. Karilahti, Defect and yield analysis of semiconductor components and integrated circuits, Ph.D. thesis, Helsinki University of Technology, Espoo, Finland, February 2003.

[2] D. H. Macdonald, Recombination and trapping in multicrystalline silicon solar cells, Ph.D. thesis, Department of Engineering, The Australian National University, Canberra, Australia, May 2001.

[3] M. Spiegel, Microwave induced remote hydrogen plasma (MIRHP) passivation of multicrystalline silicon solar cells, Ph.D. thesis, Konstanz University of Physics, Konstanz, Germany, October 1998.

[4] E. B. Yakimov, "Dislocation-point defect interaction effect on local electrical properties of semiconductors," Journal de Physique III France, vol. 7, no. 12, pp. 2293-2307, 1997.

[5] M. S. Kulkarni, J. Libbert, S. Keltner, and L. Muléstagno, "A theoretical and experimental analysis of macrodecoration of defects in monocrystalline silicon," Journal of the Electrochemical Society, vol. 149, no. 2, pp. G153-G165, 2002.

[6] G. E. McGuire, Ed., Semiconductor Materials and Process Technology Handbook, William Andrew/Noyes, Park Ridge, NJ, USA, 1988.

[7] W. C. Dash, "Copper precipitation on dislocations in silicon," Journal of Applied Physics, vol. 27, no. 10, pp. 1193-1195, 1956.

[8] E. Sirtl and A. Z. Adler, "Chromic-hydrofluoric acid as a specific system for the development of Etch pits on silicon," Zeitschrift für Metallkunde, vol. 52, no. 8, pp. 529-531, 1961.

[9] F. Secco d'Aragona, "Dislocation Etch for (100) planes in silicon," Journal of the Electrochemical Society, vol. 119, no. 7, pp. 948-951, 1972.

[10] K. H. Yang, "An Etch for delineation of defects in silicon," Journal of the Electrochemical Society, vol. 131, no. 5, pp. 11401145, 1984.

[11] K. H. Yang, "A preferential Etch for silicon crystals," in Semiconductor Processing, ASTM STP 850, D. C. Gupta, Ed., American Society for Testing and Materials, Philadelphia, Pa, USA, 1984.

[12] W. M. Jenkins, "A new preferential Etch for defects in silicon crystals," Journal of the Electrochemical Society, vol. 124, no. 5, pp. 479-762, 1977.

[13] V. G. Popov, "Semiconductor physics," Quantum Electronics \& Optoelectronics, vol. 3, no. 4, pp. 479-488, 2000.

[14] S. Pizzini, "Chemistry and physics of segregation of impurities at extended defects in silicon," Physica Status Solidi (a), vol. 171, no. 1, pp. 123-132, 1999. 


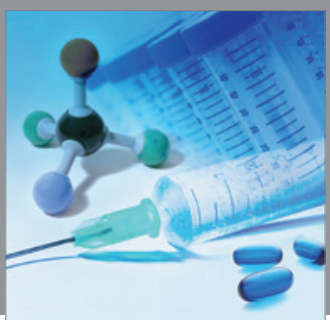

International Journal of

Medicinal Chemistry

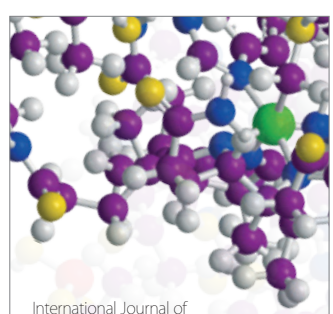

Carbohydrate Chemistry

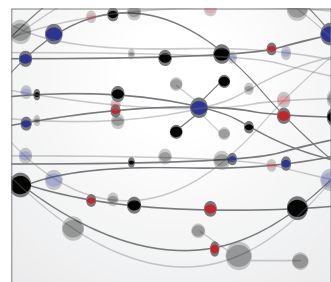

The Scientific World Journal
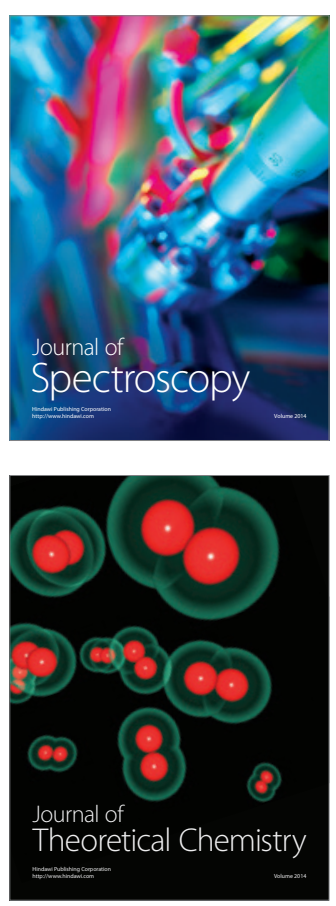
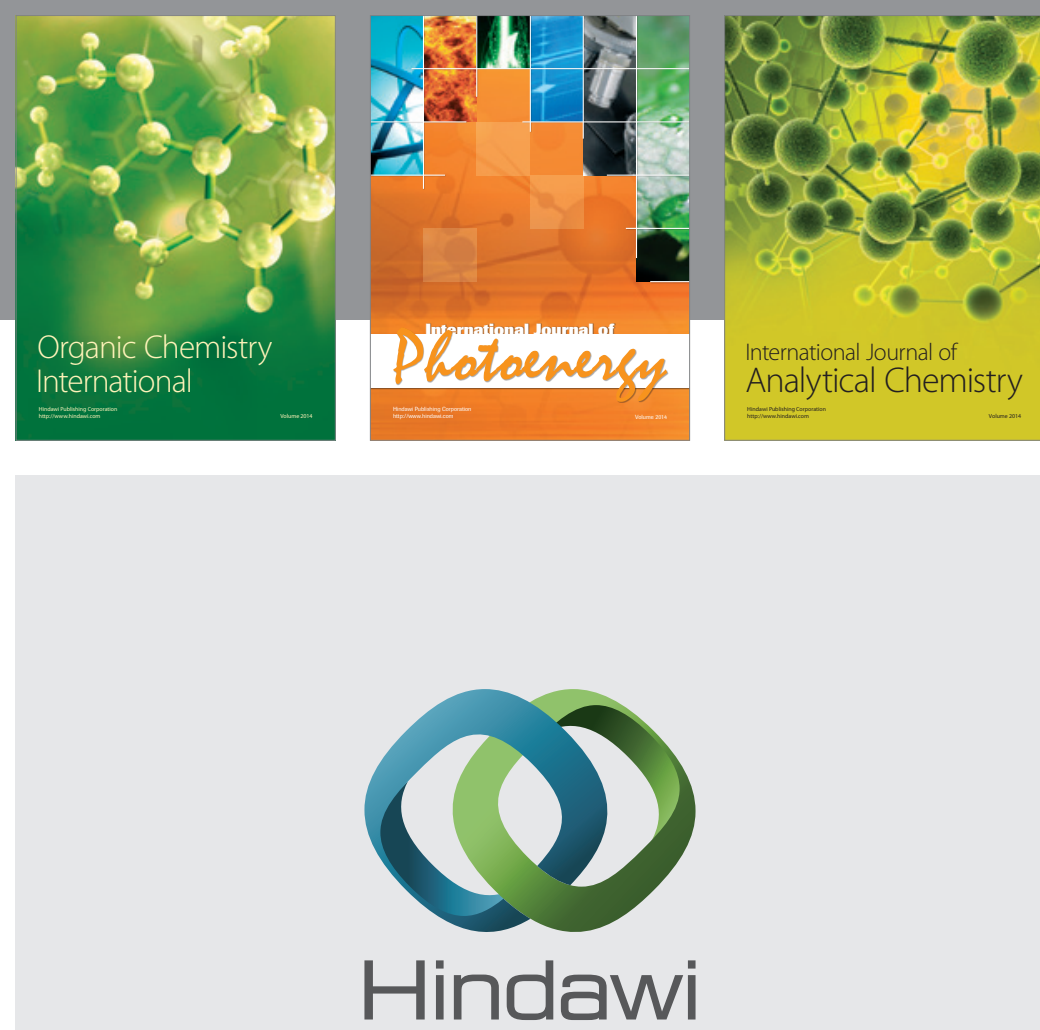

Submit your manuscripts at

http://www.hindawi.com
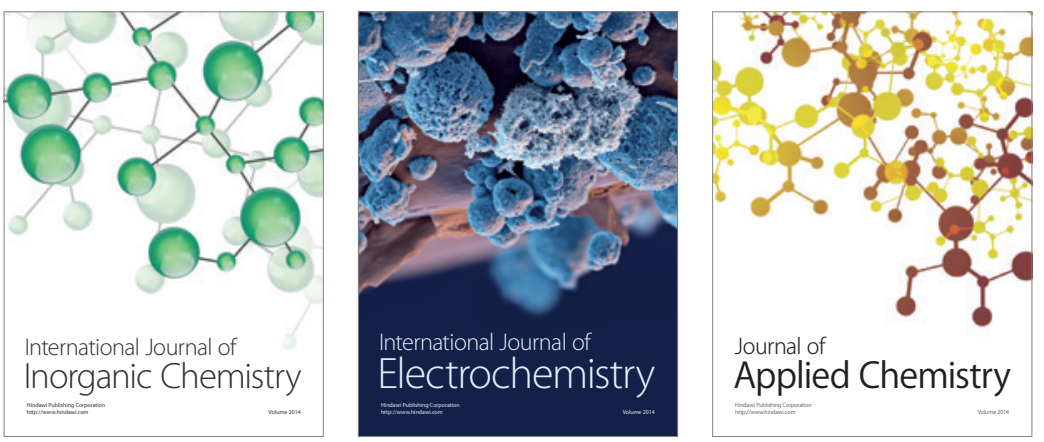

Journal of

Applied Chemistry
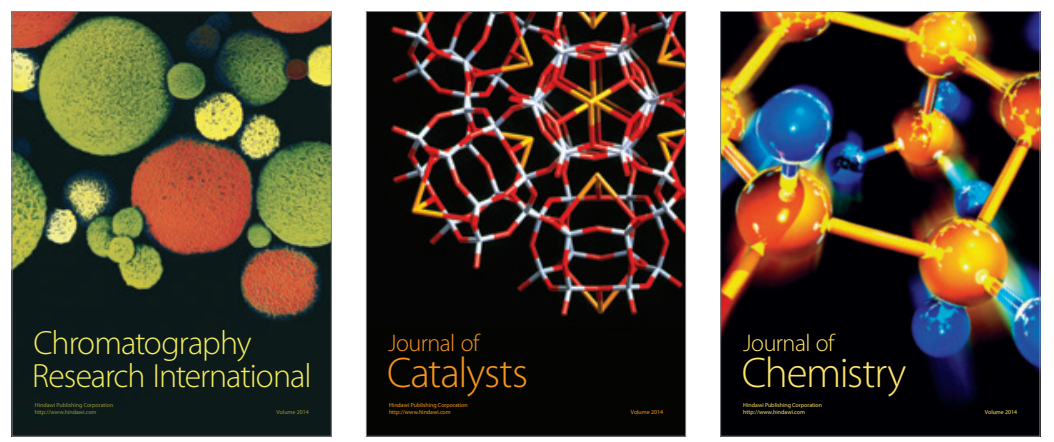
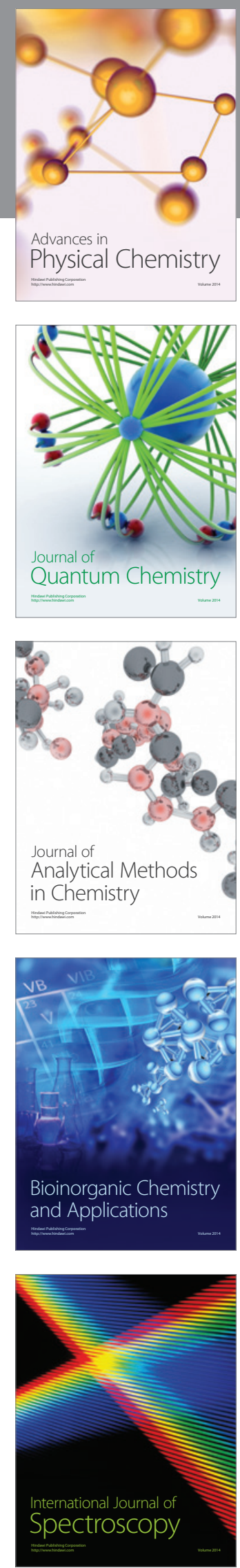